\title{
General Purpose Pulse Shape Analysis for Fast Scintillators implemented in Digital Readout Electronics
}

Abstract-Pulse shape discrimination applied to certain fast scintillators is usually performed offline. In sufficiently high-event rate environments data transfer and storage become problematic, which suggests a different analysis approach. In response, we

\section{S. J. Asztalos, W. Hennig, W. K. Warburton}

have implemented a general purpose pulse shape analysis algorithm in the XIA Pixie-500 and Pixie-500 Express digital spectrometers. In this implementation waveforms are processed in real time, reducing the pulse characteristics to a few pulse shape analysis parameters and eliminating time-consuming waveform transfer and storage. We discuss implementation of these features, their advantages, necessary trade-offs and performance. Measurements from bench top and experimental setups using fast scintillators and XIA processors are presented.

Keywords - Pulse shape discrimination; DSP; FPGA; digital signal processors

\section{Introduction}

A variety of scintillators have the ability to discern between different types of radiation, such as neutron and gamma radiation fields. This pulse shape discrimination (PSD) capability is thought to arise in organic scintillators from variations in the delayed phosphorescence in high $\mathrm{dE} / \mathrm{dx}$ events, i.e., those resulting from proton recoil [1]; in inorganic scintillators the quenching of fast fluorescence similarly modifies the pulse shape depending on particle type. As a result, neutrons produce a larger fraction of a slow scintillation light component than gammas in organic scintillators, which can be detected by suitable pulse shape analysis (PSA) methods. In addition, phoswich detectors, consisting of multiple layers of different scintillators, produce different pulse shapes depending on which layer absorbs the radiation, which can be used for particle identification [2,3,4]. Consequently, many materials and PSA algorithms have been proposed and studied in this context for many years $[5,6,7,8,9,10]$. Overall, the PSD tends to depend more on the choice of scintillator than the PSA algorithms, many of which produce similar figures of merit (FOM) $[11,12]$, though processing complexity can vary widely. In addition to the choice of scintillator and PSA algorithms, the quality of the PSD also depends on the choice of the readout electronics. Traditionally, analog systems have been used and had been shown to perform better than early digitizers sampling at $40 \mathrm{MHz}$ [13], though subsequent work demonstrated that digitizers that sample at $100 \mathrm{MHz}$ or higher outperform their analog counterparts [14]. Analog to digital converters with 12-14 bit precision are desired for best results $[15,16]$. While initially digital readout electronics has been used to simply capture 
detector waveforms for offline analysis [17, 18], in recent years detector readout electronics have become more and more

powerful, with increased digitization rate, precision, and on-board processing capabilities. The focus is thus shifting to performing PSD online in the readout electronics $[19,20,21]$. The main benefit is that it allows much higher throughput rates since waveforms do not have to be transferred from the electronics to hard drive or other storage. High throughput is of specific interest in assaying or monitoring special nuclear materials with fast scintillators [22], specifically those containing the prolific neutron emitter ${ }^{240} \mathrm{Pu}$, which emits $64 \mathrm{k}$ neutrons/s/kg. At (sustained) data rates of 10-100 MB/s with USB, PCI, or VME, transferring several hundred samples per waveform limits the throughput to $10,000-100,000$ pulses/s, and throughputs may be lower in practice due to acquisition dead times. Newer I/O standards allow higher data rates, but newer digitizers also tend to operate at higher rates and thus generate more data. In contrast, if waveforms are processed online in a digital signal processor (DSP) or field programmable gate arrays (FPGA) and only a few result values have to be transferred per pulse, throughput can be orders of magnitudes higher; and if the results can be accumulated into spectra online, throughput is only limited by the processing speed of the electronics. With proper pipelining of the processing in the FPGA, in principle true real time processing of the signal is possible, with PSA values computed for every pulse "as it comes in". However, implementation is more cumbersome, especially on FPGAs, and the algorithms can only have limited complexity (e.g. divisions are difficult except for powers of two). It is also more difficult to modify the PSA for varying conditions or applications - a minor change in FPGA logic requires recompilation of the whole device, potentially producing timing errors and glitches in supposedly unchanged areas.

We previously implemented a very specific DSP pulse shape analysis for beta/gamma coincidence detection with a phoswich detector using the Pixie-4 [22, 23], a digital data acquisition module made by XIA LLC. This code makes use of the userprogrammable DSP function calls to customize the standard operation of the module. The functions have been shown to be also applicable to neutron/gamma PSD with CLYC and liquid scintillators [24]. In this work, as a first step, we have implemented a more general purpose version in the DSP of the Pixie-500 [25] and, as a second step, in the FPGA and DSP of the Pixie-500 Express. The new firmwares were then tested in several applications and compared to offline processing results. The approach used here is a digital version of the Charge Comparison Method [3 4], where two sums over characteristic regions of the pulse are accumulated and a suitable ratio expresses the difference in pulse shape. This is a well-established method used in a variety of applications, well suited for online processing due to its simplicity.

The Pixie-500 and Pixie-500 Express are 4-channel digital data acquisition modules based on the PXI and PXIe standards, respectively. A small analog section adjusts the incoming signal in gain and offset to match the input range of an analog to digital converter (ADC), and includes a Nyquist filter to limit the bandwidth to roughly half the digitization rate (resulting in a minimum rise time of 2-3 samples). The digital data stream from the ADC is processed in an FPGA for triggering, pile-up 
inspection, and filtering in real time, and optionally waveforms can be captured for offline analysis. A DSP performs event-byevent post processing with more complex operations, such as corrections for the exponential decay of the pulses. Pulse heights are histogrammed in on-board memory. The Pixie-500 Express digitizes at $500 \mathrm{MHz}$ with 14 bit precision (Pixie-500: 12 bit), and uses a 300 MHz 32 bit floating point DSP (Pixie-500: 75 MHz 16 bit fixed point). The Pixie-500 Express data connection to the host PC is via an x4 PCI Express link (Pixie-500: 32bit PCI) that allows readout rates of several hundred MB/s, in parallel for each module in the chassis (Pixie-500: 100 MB per chassis). To eliminate acquisition dead time, the Pixie-500 Express buffers results for several detected pulses in its Virtex-4 FPGA memory, so called zero dead time (ZDT) buffers, because the only remaining dead time is due to the minimum pulse separation enforced by the pile-up inspection to be able to measure 2 pulses without interference. When pileup inspection is disabled, closely following pulses and overlapping waveforms can be recorded.

\section{Firmware Implementation}

\subsection{DSP Implementation with Pixie-500}

As part of the event-by-event processing, the Pixie-500's DSP can read captured waveforms into local memory and execute user programmable functions. In this case, the functions compute baseline average sum B, amplitude A, two sums Q0 and Q1 over characteristic areas of the pulse, and rise time RT, as shown in Figure 1. The first 8-16 samples of the waveform are summed and normalized to obtain B. The maximum sample in the waveform is located by a sample by sample search in a waveform sub region defined by the user prior to data acquisition (to reduce the processing time), then subtracted by B to obtain A. From the maximum towards earlier points, the waveform is then searched for the samples before and after the $10 \%$ and $90 \%$ constant fraction level of A. Four input parameters (L0, L1, S0, S1), specified prior to the data acquisition, define the length and position of the sums Q0 and Q1 relative to the 10\% threshold, as shown in Fig. 1. The sums Q0 and Q1 are baseline subtracted by B scaled according to their length L0 and L1, respectively. The rise time is the time difference between the $10 \%$ and $90 \%$ level of the pulse, computed with linear interpolation from the ADC samples just below and just above each threshold to a precision of 1/16 of a sample. Intermediate steps are performed using 32 bit words to retain precision before downshifting to extract the final result. A sixth return value, or PSA ratio, can be selected by the user to best match a specific application. In what follows we generally use the ratio $\mathrm{R} 1=\mathrm{Q} 1 / \mathrm{Q} 0$, but other ratios have been implemented as well. All told, these data, plus the timestamp and overall pulse height E computed with a trapezoidal filter, are written into an event header, followed by optional storage of the full waveform. From the number of instructions in the DSP code, we compute the throughput limit to be $\sim 45,000$ pulses/s for 150 samples per waveform, and roughly inverse proportional to the number of samples in the waveform. In comparison, reading full waveform data through the PCI interface limits the throughput to 1,000 to 5,000 pulses/s. 


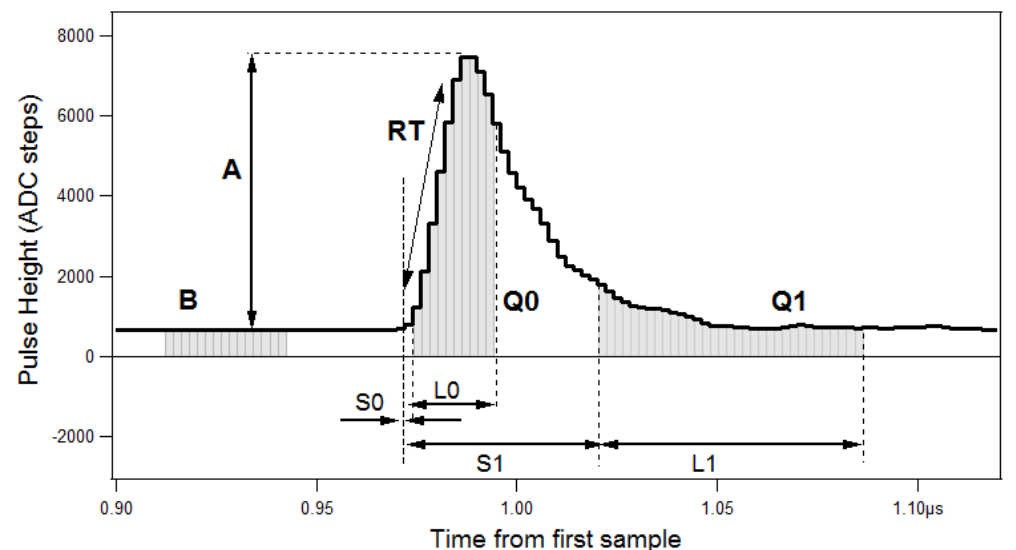

Figure 1 PSA input and output parameters. The DSP or FPGA compute baseline B, amplitude A, rise time RT and sums Q0 and Q1 using user defined delays from trigger point S0, S1 and lengths L0 L1.

\subsection{FPGA Implementation with Pixie-500 Express}

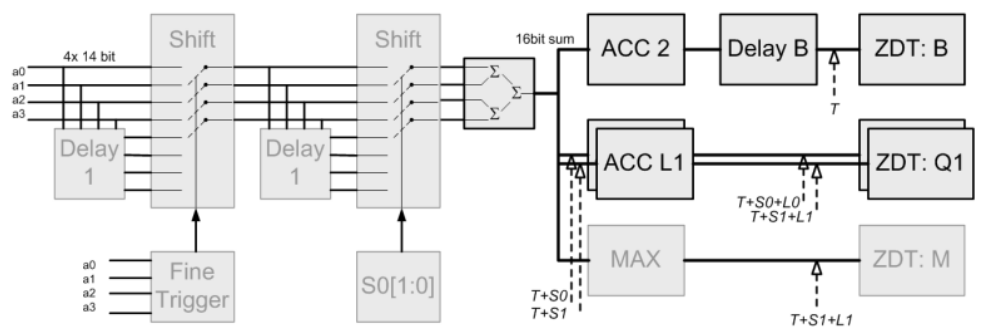

Figure 2 Block diagram of FPGA implementation described in the text. Gray boxes are only present in implementation FPGA_2.

Modern FPGAs can accept very high rate input signals, but internal processing is limited by logic and routing delays to rates of roughly $200 \mathrm{MHz}$ (For example, an N-bit adder requires for each bit the carry from the previous bit sum, so that the end result is available only after $\mathrm{N} \sim 0.1 \mathrm{~ns}$ logic operations plus routing delays). In the Pixie-500 Express, the 14 bit, $500 \mathrm{MHz}$ data stream from the $\mathrm{ADC}$ is therefore processed in sets of 4 samples at $125 \mathrm{MHz}$, for example, to generate a coarse trigger $\mathrm{T}$ at the rising edge of a pulse with a precision of $8 \mathrm{~ns}$ (see Fig.2). For the PSA functions in the FPGA, a 4-sample sum of incoming data is thus the basic building block for the PSA sums. The 4-sample sum is formed with 2 adders summing 2 samples, each in one processing cycle, and a third adder summing the 2 -sample sums in the following processing cycle. The resulting 4-sample sum is thus updated every processing cycle, but is 2 cycles delayed to the incoming data. For simplicity, the base length is fixed to 8 samples, and the base sum is computed continuously as the sum of two 4-sample sums, latched with appropriate delay into a ZDT output buffer by the trigger. In a first implementation (referred to below as FPGA_1), the PSA sums Qi were limited a) to start with a fixed delay Si relative to the coarse trigger, b) to have delays Si of multiples of 4 samples, and c) to have lengths $\mathrm{Li}$ 
103 of multiples of 4 samples. At every trigger, the process of summing is started by clearing the sum register and loading a counter 104 with $(\mathrm{Li}+\mathrm{Si}) / 4$. When the counter reaches $\mathrm{Li} / 4$ the 4 -sample sums are added to the PSA sum until the counter reaches 0 . The PSA 105 sum is latched into a ZDT output buffer by a delayed signal of the coarse trigger. Two such summing circuits are implemented 106 for the two sums, allowing their length and delay to be set independently. In a subsequent revision (FPGA_2), limitations a) and 107 b) were removed. This required complex logic for processing the 4 samples within each set, often 4 circuits in parallel, and was 108 not applied to limitation c) since that was found to be not unduly restrictive in practice. For a), a separate leading edge trigger 109 was implemented for each of the 4 samples in a set. The 4-sample sum was assembled from 4 samples out of the current and 110 previous set depending on which sample triggered first, i.e. shifting the 4-sample sum forward 0-3 samples if sample 0-3 111 triggered first, respectively. For b), a second such shifter was added, based on the lowest 2 bits of the user specified delay. In 112 addition, peak capture logic was added to record the maximum value of the signal during $(\mathrm{L} 1+\mathrm{S} 1) / 4$, the longer and later of the 113 two sums. In this firmware implementation mode the DSP is relegated to reading out the base sum B, the two PSA sums, and the 114 maximum from the FPGA ZDT buffers as part of the overall event processing, then subtracting B from the PSA sums and the 115 maximum to compute A, Q0, and Q1. In FPGA_2, the DSP also computes 1000 x R1, the factor 1000 introduced to provide 116 more significant digits than available with integers. Other ratios can relatively easily be added by modifying only the DSP code. 117 Preliminary tests indicate the throughput limit to be above 500,000 pulses/s, mainly depending on the time spent by the DSP to 118 read raw PSA values from the FPGA and write the end results into the output data stream (but not on the waveform length). 119 Currently no CFD trigger or rise time computation is implemented in the FPGA.

\section{Results and Discussion}

\subsection{Pulser Tests}

We first tested the online PSA implementations under idealized conditions using a pulser signal. A mixed neutron/gamma 124 field was simulated by programming the FPGA of an XIA PXI-PDM (pulser) to produce a digital data stream of randomly 125 triggered pulses with differing rise and decay times but identical amplitudes, which are output as analog signals via an on-board digital to analog converter. 


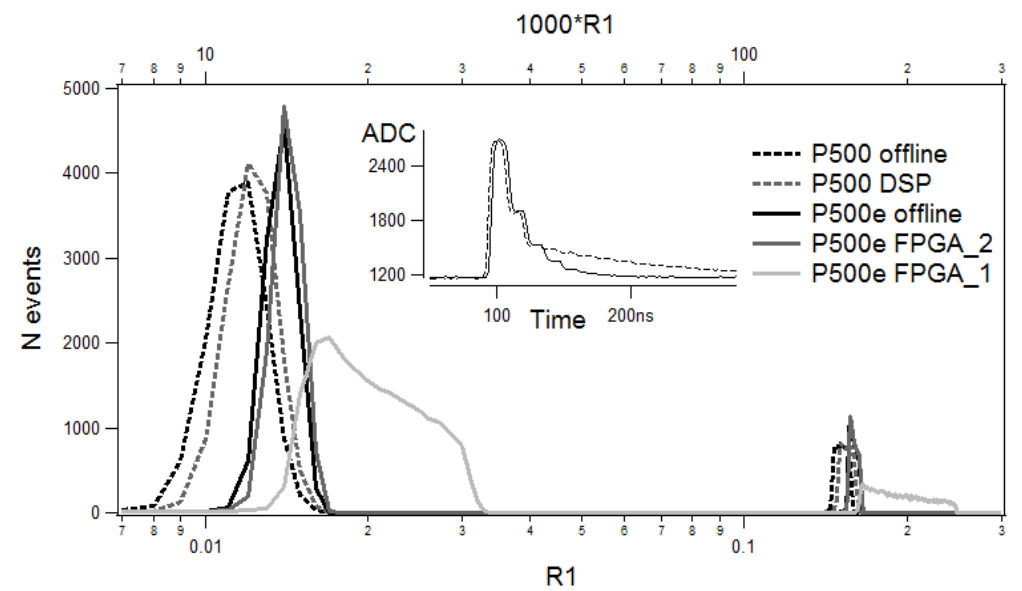

Figure 3 A comparison of PSA ratios for offline versus online data processing using a fixed amplitude pulser for the input signal. The lower peaks correspond to gamma-like signals (solid line in inset), the upper ones to neutron-like signals (dashed). The text describes the various acquisition modes.

List mode data was acquired with the Pixie-500 Express (online FPGA_1, online FPGA_2 and offline) and the Pixie-500 (online DSP and offline). For online methods, R1 was computed in the respective DSP as described above, whereas for the offline methods, R1 was computed by equivalent algorithms in IGOR Pro. For each method, R1 values were histogrammed for 20,000-50,000 pulser events as shown in Fig. 3. The Pixie-500 DSP (dashed gray) and offline (dashed black) give very close results, as expected. The Pixie-500 Express first FPGA implementation (FPGA1, solid light gray) performs poorly, producing broad, asymmetric peaks. The second FPGA implementation (FPGA_2, solid dark gray) performs the best of all approaches, producing very narrow peaks and nearly identical to the offline computation (solid black). Clearly an FPGA-based approach to PSA, if implemented carefully, is competitive with other approaches, while obviating the need for waveform capture and storage. In the following, we explore whether this performance is maintained under more realistic conditions. We continue to write waveforms to disk only so that we may directly compare the same data set.

\subsection{EJ-309 neutron/gamma PSD}

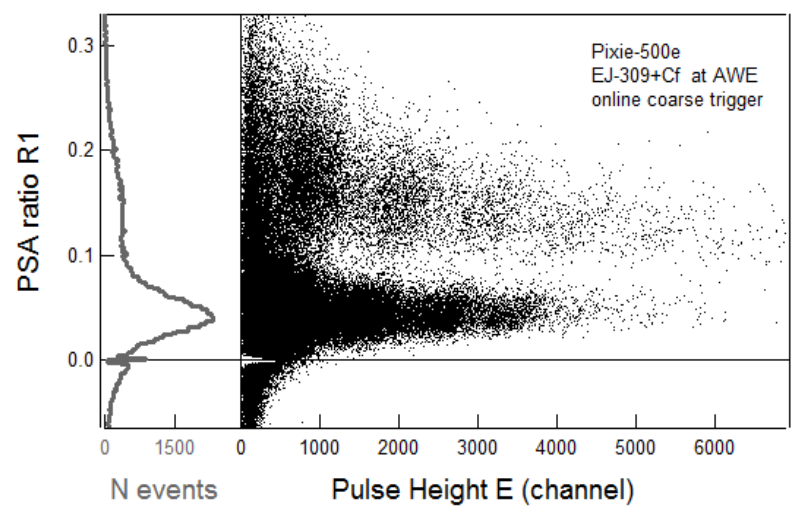



energy were computed online with the Pixie-500 Express in the FPGA with the 4-sample coarse triggering. The ratio was computed offline.

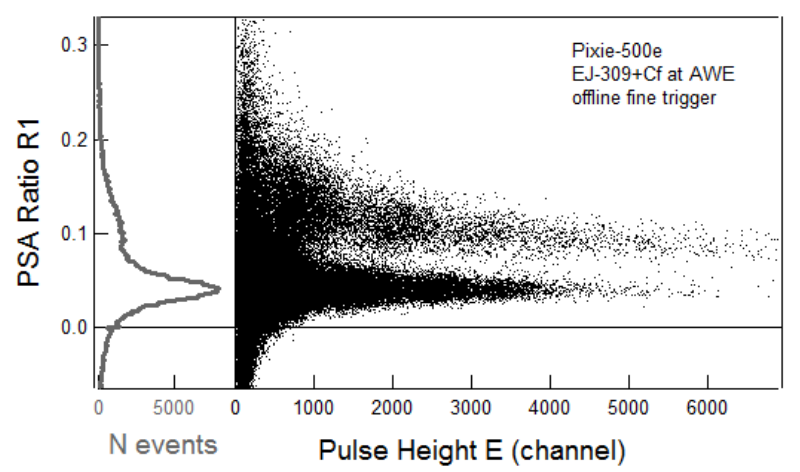

Figure 5 PSA scatter plot for neutron/gamma separation from an EJ-309 scintillator and a ${ }^{252} \mathrm{Cf}$ source, computed

The Pixie-500 Express firmware (FPGA_1) was tested with the signal from several channels of a 12-detector array of liquid scintillators (EJ-309, Eljen Technologies) at AWE Plc. A ${ }^{252} \mathrm{Cf}$ source was placed at the center of the array. The Pixie-500 Express captured waveforms for offline analysis and at the same time computed the PSA sums in the FPGA. The PSA parameters were set to $\mathrm{L} 0 / \mathrm{L} 1 / \mathrm{S} 0 / \mathrm{S} 1=20 / 40 / 0 / 36$ after brief trail-and-error for optimizing the separation of the gamma and neutron branches. Fig. 4 shows the online results plotted as R1 vs E, with the ratio computed from the sums during the plotting process, and the histogram of R1 (projection to left axis). Neutrons and gammas form two branches in the scatter plot, as commonly observed in such experiments. The captured waveforms were then processed offline for comparison. The offline processing allowed variation of PSA sum lengths and positions, as well as the different trigger definitions to detect the rising edge of the pulse. The scatter plot for $\mathrm{L} 0 / \mathrm{L} 1 / \mathrm{S} 0 / \mathrm{S} 1=20 / 40 / 0 / 36$ is shown in Fig. 5, using a "fine" trigger regarding every sample, not every 4th. The offline computation produces visibly narrower distributions for the gamma and neutron branches.

To quantify the differences, we use the standard definition of the FoM, defined as $(\mathrm{Pn}-\mathrm{Pg}) /(\mathrm{Sn}+\mathrm{Sg})$, where $\mathrm{Pn}$ is the peak of the neutron distribution, and Sn the FWHM of the neutron peak, and similarly for the gamma peak (Fig. 6). All events down to the minimum recorded pulse height are included. For (coarse trigger) online processing, the FoM is $0.38 \pm 0.04$ compared to $0.65 \pm 0.04$ for offline processing with the fine trigger. (A major source of FoM uncertainty is the fitting of the rather poorly resolved double peak in the histogram.) When shifting the trigger position in online processing by $+/-3$ samples (gray triangles in Fig. 6), it can be seen that a late trigger significantly reduces FoM, emphasizing the importance of the first few samples on the rising edge of the pulse. As the coarse online trigger effectively jitters by +/-3 samples (horizontal error bars in Fig. 6) due to the random position of the rising edge within a set of 4 samples, its overall FoM is essentially an average of that range. Purposely 

trigger (gray diamonds in Fig. 6) results in lower FoM than the fine or coarse trigger.

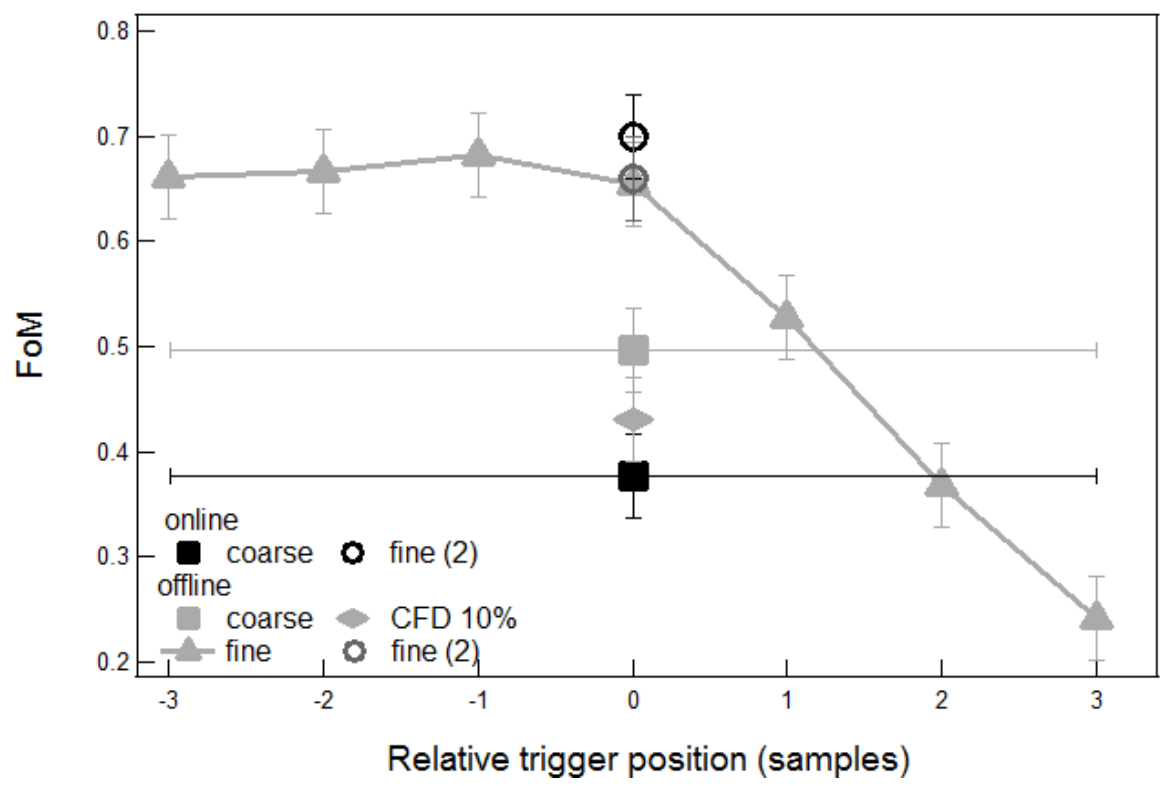

Figure 6. FOM as a function of trigger position relative to the leading edge of the pulse. Coarse triggering within a set of 4 samples is shown with error bars of $+/-3$. Fine online triggering is equal to fine offline triggering (in a second data set)

The FoM can be improved by applying a low energy cutoff to the data. A cutoff of $\sim 200 \mathrm{keV}$ (removing $40 \%$ of the events) is required to make the coarse offline FoM equal to the offline fine FOM (0.67). FoM values for neutron/gamma discrimination reported in the literature fall in the range of $\sim 1.0$ to $\sim 2.0[16,27]$ for EJ-309 and $\sim 1.0$ to $\sim 2.7$ for EJ-301 [28], but are often given for a narrow energy range, and are found to vary by source, geometry, as well as digitization precision and rate [16, 28] (the latter implying a certain signal bandwidth). PSD parameter definition may also vary from R1 used here, which makes meaningful comparison difficult. When the PSA parameters were optimized offline, the largest FoM for our data was $1.14 \pm 0.04$ for L0/L1/S0/S1 = 8/40/0/16. Very broad optima were observed, in particular for variations in length. Based on this result, we found it unnecessary to remove the limitation of PSA length being a multiple of 4. Finer online triggering is however highly desirable, hence the second implementation of the FPGA firmware. A second data set acquired with implementation FPGA_2 achieved equal FoM for fine online and fine offline triggering (open circles in Fig. 6)

\subsection{Stilbene gamma/alpha PSD}

A material more recently developed for pulse shape analysis is crystal Stilbene [29]. While the main purpose of the materials development is neutron/gamma discrimination, in our work we explored it as a gamma/alpha discriminator. Being a solid rather 
than a liquid, it requires no container, and so an alpha source can easily be brought in close enough contact with the scintillator. In this test, a small flat crystal of Stilbene (Inrad Optics, approx. $2 \mathrm{~mm}$ x $2 \mathrm{~cm} \mathrm{x} 1 \mathrm{~cm}$ ) was coupled to a 1" PMT (Hamamatsu) with optical grease. The other side was covered with 4-8 layers of Teflon tape. A small gap was left in the taped area, over which a ${ }^{241} \mathrm{Am}$ source was placed, emitting alphas and photons. The whole PMT/scintillator/source arrangement was then placed in a light tight box. The PMT is biased to $~ 950$ V with an iseg EHQ105M HV module and read out by a Pixie-500 or Pixie-500 Express which performed the PSA (DSP or FPGA_2) and captured waveforms for offline comparison. A ${ }^{137}$ Cs source was put on the outside of the box to increase the photon rate.

199 PSA scatter plots for online and offline computations are shown in Fig. 7Figure for optimized parameter settings L0/L1/S0/S1 $200=12 / 64 / 0 / 32$. Alphas excite more of the slow scintillation process and therefore have relatively larger Q1, populating the upper group of events in the scatter plots. Online and offline scatter plots for the Pixie-500 Express (using the same data) look almost identical. In the online data, "bad" events (often with negative values due to noise spikes in the baseline) appear at 64K, a total of 812 out of $\sim 470,000$ in this data set. In the offline data, 827 events are "bad" and their negative ratios do not appear on the log scale graph. Coarseness of integer numbers becomes visible for R1 $<10$ in online computation, but this affects only a small minority of counts.
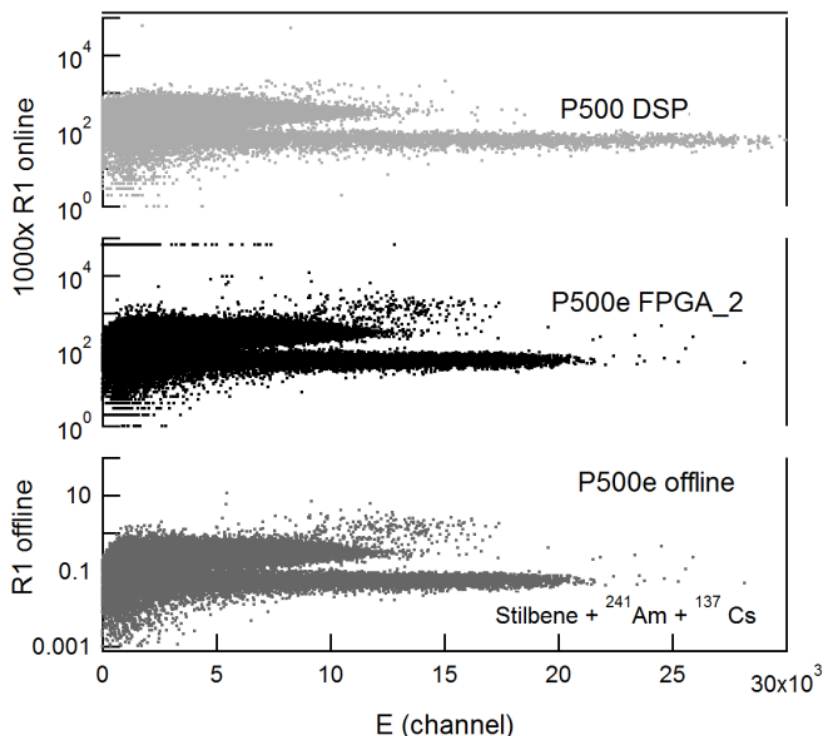

Figure 7 Scatter plots of R1 vs E for Stilbene with Pixie-500 and Pixie-500e. Pixie-500e FPGA_2 and offline results are

\section{from the same measurement.}

The data from Fig. 7Figure is histogrammed in Fig.8. For the Pixie-500 Express measurements, the FoM is 1.393 for Pixie500 Express online computation, 1.392 for Pixie-500 Express offline computation, and 1.089 for Pixie-500 DSP online computation. With clear double peaks, the uncertainties are $\sim 0.03$ or less. The Pixie-500 Express histograms are essentially 
identical (except the online implementation of the division returns negative numbers near $64 \mathrm{~K}$ and so its histogram ends at zero).

It is to be expected that the FOM for the Pixie-500 DSP online computation is close to that for the Pixie-500 Express offline computation as both methods are operating on equivalent waveforms. Yet, it is somewhat poorer than those for the Pixie-500

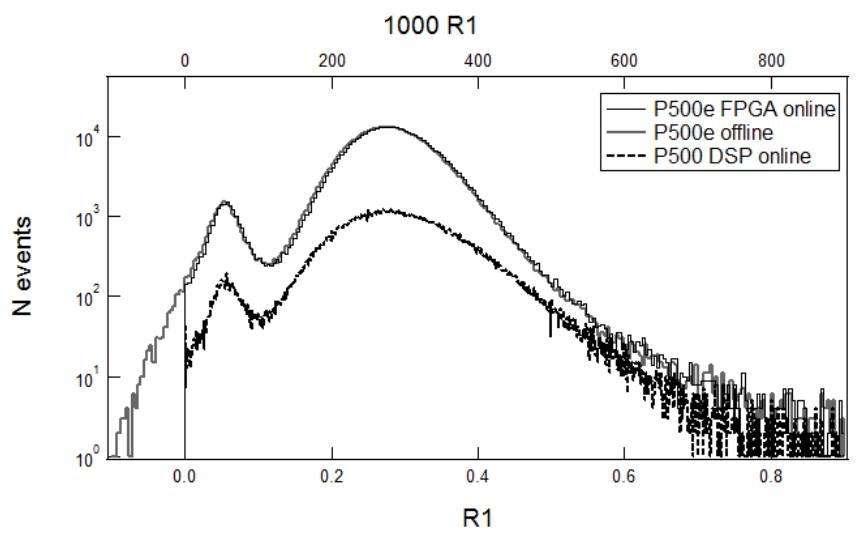

Figure 8 Histograms of PSA ratio R1 for data shown in Figure 7. Pixie-500 Express online and offline distributions are

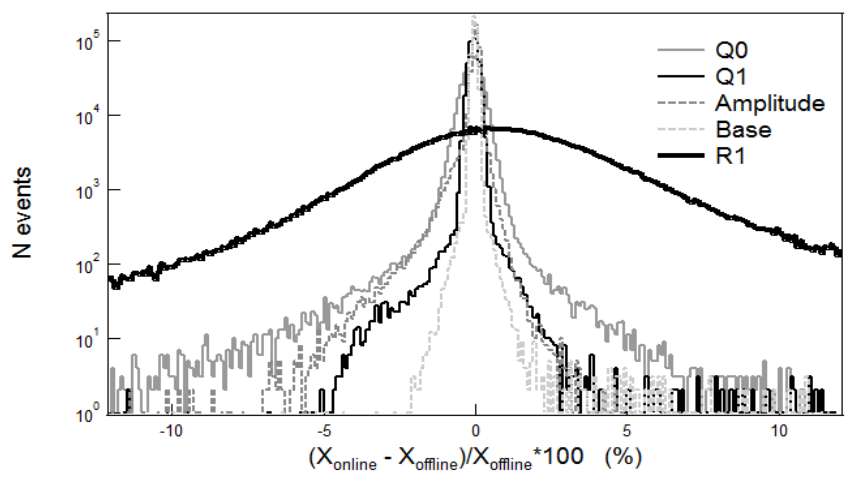

Figure 9 Histogram of normalized differences between online and offline computation of PSA results. Differences are small for amplitude, base, and Q1, larger for Q1 and PSA ratio R1.

Table 1 Quantification of difference between online and offline computation

\begin{tabular}{|c|c|c|c|}
\hline \multirow[t]{2}{*}{ Parameter } & \multicolumn{3}{|c|}{$\begin{array}{l}\text { Fraction of counts within range around } \\
\text { zero }\end{array}$} \\
\hline & $1 \%$ & $2 \%$ & $10 \%$ \\
\hline $\mathrm{Q} 0 *$ & 0.976 & 0.991 & 0.998 \\
\hline Q1* & 0.996 & 0.998 & 1 \\
\hline Amplitude & 0.985 & 0.995 & 0.998 \\
\hline Base & 0.999 & 1 & 1 \\
\hline Q1/Q0 & 0.274 & 0.495 & 0.947 \\
\hline
\end{tabular}

* Not baseline subtracted

It is also instructive to compare the differences in Pixie-500 Express online and offline results for each individual parameter. 
To do so, we compute the difference $\left(\mathrm{X}_{\text {online }}-\mathrm{X}_{\text {offline }}\right) / \mathrm{X}_{\text {offline }}$ for each pulse, $(\mathrm{X}$ stands for Q0, Q1, $\mathrm{A}, \mathrm{B}$, or R1) and accumulate it in a histogram for all pulses in the data set. Q0 and Q1 here are not baseline subtracted to avoid addition of error from the baseline. The histograms are shown in Fig. 9. Base and Q1 differ very little, as the signal changes very slowly in the baseline and the falling portion of the pulse. The amplitude differs slightly more, however since the maximum is found accurately without depending on trigger position, the baseline subtraction adds the majority of differences. Q0 differs more, due to the fast rising pulses have less than $1 \%$ difference in Q0, Q1, amplitude or base, and $295 \%$ of pulses have less than $10 \%$ difference in R1, see

\subsection{CsI/Stilbene alpha/beta/gamma PSD}

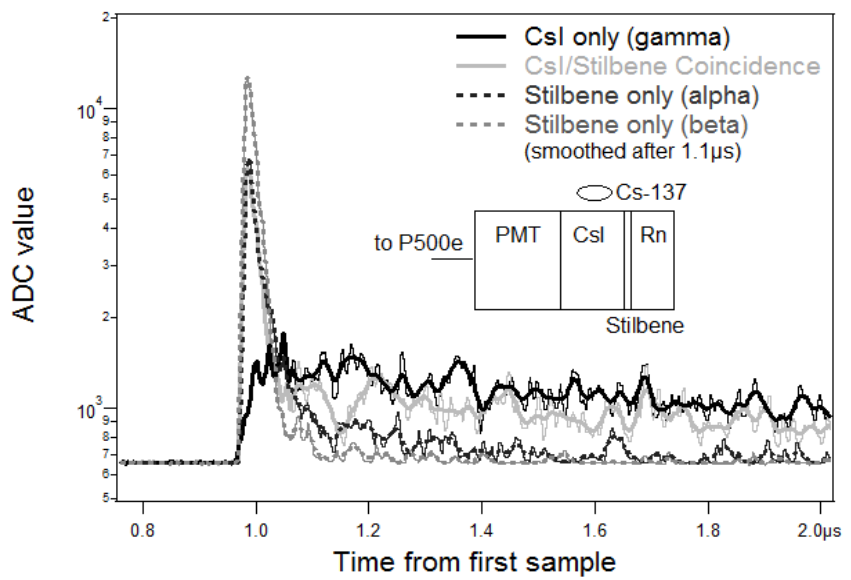

Figure 10 Waveforms from CsI/Stilbene phoswich detector. 4 different types can be identified, as described in the main text. Waveforms are smoothed after $1.1 \mu$ for clarity.

The Pixie-500 Express firmware (FPGA_1) was tested also with a phoswich detector for detection of radioactive gases. Originally designed to study $\mathrm{Al}_{2} \mathrm{O}_{3}$ coating of BC-404 scintillators to reduce diffusion of radioxenon [30], it was now assembled as a $1 \mathrm{~mm}$ thick x $50.8 \mathrm{~mm}$ diameter crystal Stilbene disc (Inrad Optics) optically coupled to a $50.8 \mathrm{~mm}$ tall x $50.8 \mathrm{~mm}$ diameter CsI(Tl) crystal, read out by a single PMT (ET Enterprises KB9266). The Stilbene is one end of an otherwise Aluminum cell holding radioactive gas samples. Exposed to a ${ }^{222} \mathrm{Rn}$ source (with daughters), we observe 4 types of signals from this detector shown in Fig. 10: 1) gammas interacting in the CsI, 2) betas (or gammas) interacting in the Stilbene, 3) alphas interacting in the Stilbene, and 4) gamma/beta coincidences in CsI and Stilbene (or gammas scattering between CsI and Stilbene). Gammas interacting in the CsI generate a slow rising pulse with $\sim 1$ us decay. Betas and gammas interacting in Stilbene generate the same pulse shape, a fast rise followed by a fast decay. Alphas generate a pulse shape with a fast rise and a slightly slower decay, due to a larger contribution of the slow decay component. CsI/Stilbene coincidences generate a mixture of the respective pure pulses, 

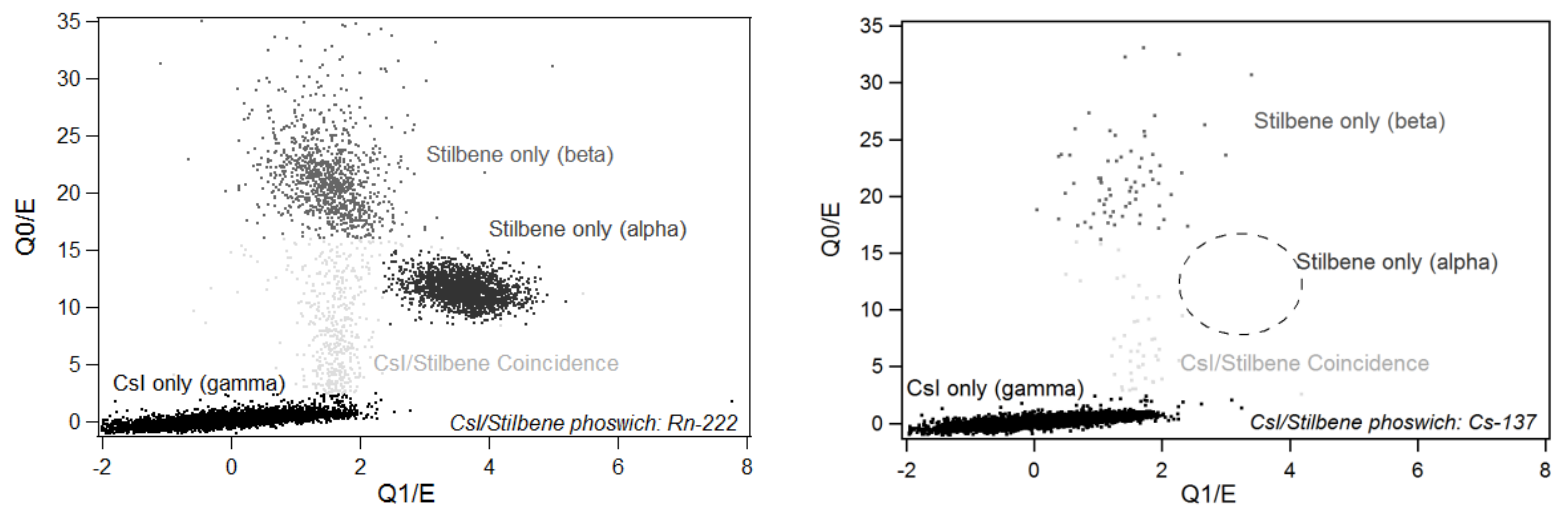

Figure 11 PSA scatter plot of Q0/E vs Q1/E for the CsI/Stilbene phoswich detector, separating alphas, betas, gammas, and beta/gamma coincidences from a ${ }^{222} \mathrm{Rn}$ source (left). With a ${ }^{137} \mathrm{Cs}$ source, no alpha events are detected.

filter. The ratios Q0/E and Q1/E were computed offline and are plotted in Fig. 11 (left). The 4 event types fall into well separated groups, with CsI/Stilbene coincidences connecting the groups of pure CsI events and pure Stilbene beta (or gamma) events. In contrast, exposing the detector to an external ${ }^{137} \mathrm{Cs}$ source (no alphas) results in a similar plot, but without the alpha events, Fig. 11 (right). The detector and PSA thus allow a 2-layer phoswich to detect alphas, betas and gammas, while in the past 3-layers have been used for such purposes [2].

In this case, a FoM defined as in the above applications would be less meaningful, since there are more than 2 event types. With appropriate regions of interest applied to the distribution in Figure 10, the pulse heights could be histogrammed separately for the different even types, to obtain pure gamma, beta, and alpha spectra from the same measurement. We note that while digitization at $500 \mathrm{MHz}$ is helpful in distinguishing Stilbene alphas and betas, the larger noise associated with the high bandwidth and the sensitivity to the arrival of randomly emitted photons complicates the analysis of CsI pulses, e.g. by making it harder to trigger reliably on small pulses.

\section{Conclusions}

In summary, we have implemented a general purpose PSA analysis in firmware and have compared the results with those from offline implementations using a pulser and a combination of sources and fast scintillators capable of discriminating between radiation fields. Broadly speaking, even the first FPGA implementation is useful in some applications, and the DSP and the second firmware implementations (with CFD or fine triggering), provided nearly equivalent results to their offline counterparts -the overall scatter plots, histograms, and FoMs are practically identical. This demonstrates that a general purpose PSA FPGA implementation is feasible and readily adaptable to other fast scintillators, while allowing 1-2 orders of magnitude higher 
throughputs. Implementation in the DSP is comparatively easier, since sequential programming is used, but processing is slower, especially where sample by sample operations on buffered waveforms are performed. Implementation in the FPGA is more complex and requires great care to debug errors and adapt to device limitations, but processing throughput is much faster. In practice, a combination of FPGA, DSP and offline processing seems to be the most rewarding - the FPGA performing the simpler, but time consuming operations such as summing and finding maxima, while computation of ratios and acceptance decisions can be performed by a DSP or offline. This also allows more flexibility for varying PSD criteria in different applications. One simple extension of this work would be the introduction of a threshold for accepting events (e.g., those above a certain PSA ratio threshold) and building neutron and gamma histograms by the DSP in on-board memory.

\section{Acknowledgements}

This work was supported in part by the US Department of Energy under awards DE-NA0001522 and NA000486-DE. We greatly appreciate discussions on PSD requirements and assistance with test measurements by L. Jones and N. McMillan, AWE Plc, UK.

\section{References}

[1] J.B Birks, Proc Phys. Soc. A64, 874 (1951)

[2] Travis L. White, William H. Miller, "A triple-crystal phoswich detector with digital pulse shape discrimination for alpha/beta/gamma spectroscopy”, NIM A 422 (1999) 144-147

[3] O. Tengblad et al, " $\mathrm{LaBr} 3(\mathrm{Ce}): \mathrm{LaCl} 3(\mathrm{Ce})$ Phoswich with pulse shape analysis for high energy gamma-ray and proton identification", NIM A 704 (2013) 19-26

[4] J.H. Ely, C.E. Aalseth, J.I. McIntyre, "Novel beta-gamma coincidence measurements using phoswich detectors", J. Radioanal. Nucl. Chem., Vol. 263, No 1 (2005) 245-250

[5] F.D. Brooks, Nucl. Instr. and Meth. 4 (1959) 151.

[6] C.L Morris et al. “A Digital Technique for Neutron-Gamma Pulse shape Discrimination”, NIM (37), pp 397-398 (1976)

[7] J.M. Adams, G. White, Nucl. Instr. and Meth. A 156 (1978) 459.

[8] D.W. Lee n, L.C. Stonehill, A. Klimenko, J.R. Terry, S.R. Tornga,” Pulse-shape analysis of Cs2LiYCl6:Ce scintillator for neutron and gamma-ray discrimination “, NIM A 664 (2012) 1-5

[9] J.P. Malkar and P.K. Kunte, "Pulse shape discrimination technique in a NaI(Ti)-CsI(Na) x-ray detector", NIM 202 (1982), $465-468$

[10] R. Folle, G. Mathe and D. Netzband, “A method for pulse shape iscrimination”, NIM 35 (1965), 250-260

[11] K.A.A.Gamage, M.J.Joyce, N.P.Hawkes, “A comparison of four different digital algorithms for pulse-shape discrimination in fast scintillators", Nuclear Instruments and Methods in Physics Research A 642 (2011) 78-83 
[12] C. Xiaohui, et al., "Analysis of three digital n/g discrimination algorithms for liquid scintillation neutron spectrometry", Radiation Measurements 49 (2013) 13-18

[13]R. Aryaeinejed, "Comparison between digital and analog pulse shape discrimination techniques for neutron and gamma ray separation”, Nuclear Science Symposium Conference Record, 2005 IEEE

[14]P.A. Soderstrom_, J. Nyberg, R. Wolters, "Digital pulse-shape discrimination of fast neutrons and g rays", Nuclear Instruments and Methods in Physics Research A 594 (2008) 79- 89

[15]C. Hellesen, M.Skiba ,G.Ericsson, E.Andersson Sundén, F.Binda, S.Conroy, J.Eriksson, M. Weiszflog, "Impact of digitization for timing and pulse shape analysis of scintillator detector signals", NIM A 720 (2013) 135-140

[16] Marek Flaska, Muhammad Faisal, David D. Wentzloff, Sara A. Pozzi, "Influence of sampling properties of fast-waveform digitizers on neutron-gamma-ray, pulse-shape discrimination for organic scintillation detectors" NIM A 729, (2013) 456-462

[17] M. Flaska, S.A. Pozzi, “Offline Pulse-Shape Discrimination Algorithms for Neutron Spectrum Unfolding”, Nuclear Science Symposium Conference Record, 2006. IEEE (Volume 2) pp. 752 - 758

[18] S.D. Jastaniah, P.J. Sellin, "Digital techniques for $\mathrm{n} / \mathrm{g}$ pulse shape discrimination and capture-gated neutron spectroscopy using liquid scintillators", NIM A 517 (2004) 202-210

[19] Shiping Li, “A real-time n/g digital pulse shape discriminator based on FPGA”, Applied Radiation and Isotopes 72 (2013) $30-34$

[20] M. Nakhostin, "Recursive algorithms for digital implementation of neutron/gamma discrimination in liquid scintillation detectors",NIM A 672 (2012), 1-5

[21] A.T. Farsoni, B. Alemayehu, A. Alhawsawi, E.M. Becker, "Real-time pulse-shape discrimination and beta-gamma coincidence detection in field-programmable gate array”, NIM A 712 (2013), 75-82

[22] A. Enqvist, M. Flaska, J. L. Dolan, D. L. Chichester, S. A. Pozzi , “A combined neutron and gamma-ray multiplicity counter based on liquid scintillation detectors" NIM A 652 (2011), 48-51

[23] W. Hennig, H. Tan, W.K. Warburton, J.I. McIntyre, “ Single Channel Beta-Gamma Coincidence Detection of Radioactive Xenon Using Digital Pulse Shape Analysis of Phoswich Detector Signals”, IEEE Trans. Nucl. Sci. 53 Vol. 2 (2006) p620

[24]W. Hennig, H. Tan, W.K. Warburton, A. Fallu-Labruyere, J.I. McIntyre, A. Gleyzer, "A phoswich well detector for radioxenon monitoring”, NIM A 579 (3), pp. 431-436. 2007

[25]S. Asztalos, W. Hennig "CLYC versus Stilbene: Optimization and comparison of two neutron-gamma discriminating scintillators", presented at the 2010 IEEE Nuclear Science Symposium, Nov 1-5, 2010, Knoxville, TN, USA.

[26]W. Hennig, S.J. Asztalos, D. Breus, K. Sabourov, W.K. Warburton, "Development of $500 \mathrm{MHz}$ Multi-Channel Readout Electronics for Fast Radiation Detectors”, IEEE Trans. Nucl. Sci., Vol. 57, No. 4 (2010), p. 2365-2370 
336 [27] I.A. Paweczak, S.A.Ouedraogo, A.M.Glenn, R.E.Wurtz, L.F.Nakae, "Studies of neutron-g pulse shape discrimination in 337 EJ-309 liquid scintillator using charge integration method, NIM A711 (2013) p21

338 [28] Cester D. M. Lunardon, G. Nebbia, L Stebanato, G. Viesta, S. Petrucci, C. Tintori, "Pulse shape discrimination with fast 339 digitizers" NIM A 748 (2014), pp. 33-38;

[29] Leslie Carman, Natalia Zaitseva, H. Paul Martinez, Benjamin Rupert, Iwona Pawelczak, Andrew Glenn, Heather Mulcahy, Roald Leif, Keith Lewis, Stephen Payne, "The effect of material purity on the optical and scintillation properties of solutiongrown trans-stilbene crystals", J Crystal Growth 368 (2013) 56-61

343 [30] W. Warburton, W. Hennig, J. Bertrand, S. George, and S. Biegalski, "Alpha-Al2O3 Diffusion Barriers to Eliminate the 344 Radioxenon Memory Effect in Phoswich Detectors", presented at the 34rd Monitoring Research Review, Albuquerque NM, USA, Sept 18-20, 2012. LA-UR-12-24325 